\title{
Dietary patterns in children: results from the Southampton Women's Survey
}

\author{
C. M. Fisk, S. R. Crozier, H. M. Inskip, K. M. Godfrey, C. Cooper, S. M. Robinson \\ and the SWS Study Group \\ MRC Epidemiology Resource Centre, University of Southampton, Southampton General Hospital, \\ Southampton SO16 6YD, UK
}

\begin{abstract}
There has been an increase in the number of studies that use dietary patterns analysis to assess diet as a whole, rather than individual foods or nutrients. Identification of dietary patterns in childhood may prove useful in investigating the relationship between early diet and health in later life. The aim of the present study was to describe the dietary patterns of 3-year-old children using principal component analysis (PCA). Data were collected from children in the Southampton Women's Survey (SWS). The SWS is a large prospective study in which a total of 12583 non-pregnant women aged 20-34 years and resident in the city of Southampton, UK were recruited between 1998 and 2002. Babies born to women in the SWS are followed up at 6 months, 12 months, 2, 3, 4, 6 and 8 years of age ${ }^{(1)}$. At age 3 years the children were visited at home where their diet, eating behaviour, physical activity and illnesses were assessed. Diet was assessed using an eighty-item FFQ, administered by trained research nurses, to record the average frequency and quantity of the foods consumed by the child over the preceding 3 months. Between 1998 and 2003, 1981 singleton babies were born to the women in the SWS. Complete dietary data were available for $1640(83 \%)$ of these children at 3 years of age.

PCA identified three distinct dietary components in the 3 year olds that explained $7.5,4.6$ and $3.7 \%$ of the total variation in the study sample. The first component was termed a 'prudent' diet pattern and was characterised by a high frequency of consumption of vegetables, fruit and juices, water, wholemeal bread and fish and low consumption of white bread, crisps, chips and processed meat. The second component was termed a 'traditional' pattern and was characterised by a high frequency of consumption of processed and red meat, puddings and green vegetables and low consumption of wholemeal bread, water and fruit juices. The third component was termed a 'convenience vegetarian' pattern and was characterised by a high consumption of vegetable dishes and vegetarian foods, beans and pulses and quiche and pizza and low consumption of meat, boiled potatoes and vegetables.

Three clear patterns of foods have been described in the diets of the children in the SWS at 3 years of age. These patterns reflect large differences in dietary choices and food consumption. The 'prudent' dietary pattern that explains the most variation in the study sample is very similar to the 'prudent' pattern that has been described previously for the women in the SWS ${ }^{(2)}$. Ongoing work is addressing the relationship between the dietary patterns of the mothers in the SWS and their children, and the influence of these patterns on growth and development in early childhood.
\end{abstract}

This work was supported by the Medical Research Council, University of Southampton, British Heart Foundation and the Food Standards Agency (contract NO5071)

1. Inskip HM, Godfrey KM, Robinson SM et al. (2006) Int J Epidemiol 35, 42-48.

2. Robinson SM, Crozier SR, Borland SE et al. (2004) Eur J Clin Nutr 58, 1174-1180. 